\title{
Building and Imaging Silicide Nanostructures in Nanowires
}

\author{
F. Panciera, Y.-C. $\mathrm{Chou}^{2,3,4}$, M. C. Reuter ${ }^{2}$, D. Zakharov ${ }^{4}$, E. A. Stach ${ }^{4}$, E. Jensen ${ }^{5}$, K. Mølhave ${ }^{5}$, S.
} Hofmann ${ }^{1}$ and F. M. Ross ${ }^{2}$

1. Department of Engineering, University of Cambridge, 9 J. J. Thomson Avenue, Cambridge, UK.

2. IBM Research Division, T. J. Watson Research Center, Yorktown Heights, NY, USA

3. Department of Electrophysics, National Chiao Tung University, 1001 University Road, Hsinchu city, Taiwan.

4. Center for Functional Nanomaterials, Brookhaven National Laboratory, Upton, NY, USA.

5. DTU-Nanotech, Department of Nano- and Microtechnology, Technical University of Denmark, Kgs. Lyngby, Denmark.

The vapour-liquid-solid (VLS) growth process allows the fabrication of semiconductor nanowires with a remarkable level of structural control. This structural versatility has contributed to the tremendous impact of VLS-grown nanowires. By tuning growth parameters and catalyst composition, we can control the composition, diameter and growth direction of the nanowires, and form branches, kinks, periodically arranged twins, and even polytype superlattices, leading to applications in low power electronics and optoelectronics. Transmission electron microscopy (TEM) has been an essential tool for imaging structures after growth, while in situ TEM has provided insights into growth mechanisms, helping to drive the development of more complex structures [1].

Here we describe a variation on the conventional VLS growth concept, developed through in situ TEM observations, which provides opportunities for building new types of nanowire-based structures. The principle is to first grow silicon nanowires using liquid AuSi catalyst droplets, then interrupt growth and supply a new species, such as a metal, that can react with the material in the droplet. In situ ultra-high vacuum TEM [1] visualizes the nature and kinetics of these droplet reactions. As one might expect, species like Ni or Co nucleate silicide phases. But, surprisingly, the phases form as faceted nanocrystals that float within the liquid droplet. Eventually each nanocrystal makes contact with its nanowire-catalyst growth interface and becomes attached. Further growth of Si allows the nanocrystal to become incorporated into the nanowire. In Figures 1 and 2, the formation and incorporation steps are imaged in situ using ultra-high vacuum TEM. Figure 3 shows a nanowire imaged after carrying out the incorporation step in an aberration-corrected environmental TEM (ETEM). The outcome can be nearperfect endotaxial incorporation of a nanocrystal of controlled structure, size and location, with the whole process repeatable to incorporate multiple nanocrystals in a single nanowire. Potential applications may include single-electron transistors, high-density memories, semiconductor lasers, and tunnel diodes.

Ultra high vacuum TEM provides reaction kinetics under well-controlled growth conditions, while aberration-corrected ETEM provides improved spatial and temporal resolution, revealing details of the phase sequence and step flow growth. However, imaging phase nucleation and growth is challenging in aberration-corrected ETEM due to its non-UHV environment and the strict stability requirement on heating. To address both issues, we describe a low-drift sample heater based on a resistively heated cantilever geometry [2], and we discuss a protocol to reduce background contamination during growth, 
based on operating the ETEM cold finger at a temperature tuned to condense water vapour but not the growth species, disilane [3]. We will show how these experimental modifications can lead to quantitative information on nanowire growth, silicide nucleation and phase transformations with high spatial and temporal resolution [4].

\section{References:}

[1] F. M. Ross, Reports on Progress in Physics 73 (2010), p. 114501.

[2] C. Kallesøe, et al., Nano Lett. 12 (2012), p. 2965.

[3] Y.-C. Chou, et al., in preparation (2015).

[4] We acknowledge the National Science Foundation (Grants No. DMR-0606395 and 0907483), ERC Grant 279342: InSituNANO, the National Science Council of Taiwan (Grant NSC-101-2112-M-009021-MY3), the Center for Interdisciplinary Science (MOE-ATU project for NCTU) and the Center for Functional Nanomaterials, Brookhaven National Laboratory, which is supported by the U.S. Department of Energy, Office of Basic Energy Sciences, under contract DE-AC02-98CH10886; C. Czarnik for assistance with image processing and A. W. Ellis for technical support.

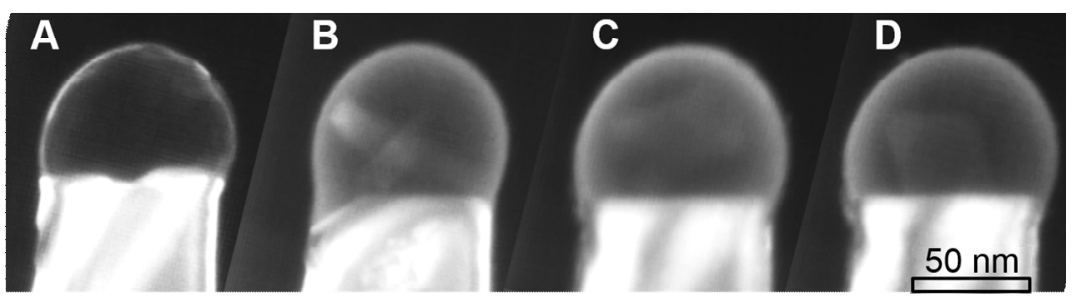

Figure 1. Formation and epitaxial contact of a $\mathrm{NiSi}_{2}$ nanocrystal in a $\mathrm{Si}$ nanowire after $1 \mathrm{~nm} \mathrm{Ni}$ deposition, oxidation to prevent sidewall reactions, then temperature increase to $500^{\circ} \mathrm{C}$ and flow of $5 \times 10^{-5}$ Torr $\mathrm{Si}_{2} \mathrm{H}_{6}$. A Below the eutectic temperature of $370^{\circ} \mathrm{C}$ the $\mathrm{Au}$ is solid. B As we exceed the eutectic temperature, AuSi liquid and Ni-silicide nanocrystals form. $\mathrm{C}$ The silicide agglomerates into an octahedral nanocrystal. D The nanocrystal attaches at the Si interface.

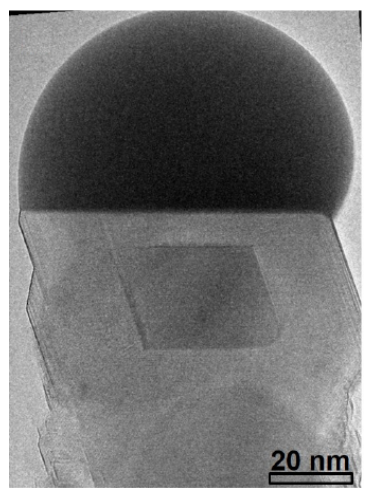

Figure 3. A nanowire with embedded $\mathrm{NiSi}_{2}$ nanocrystal, imaged after Si growth in the ETEM.

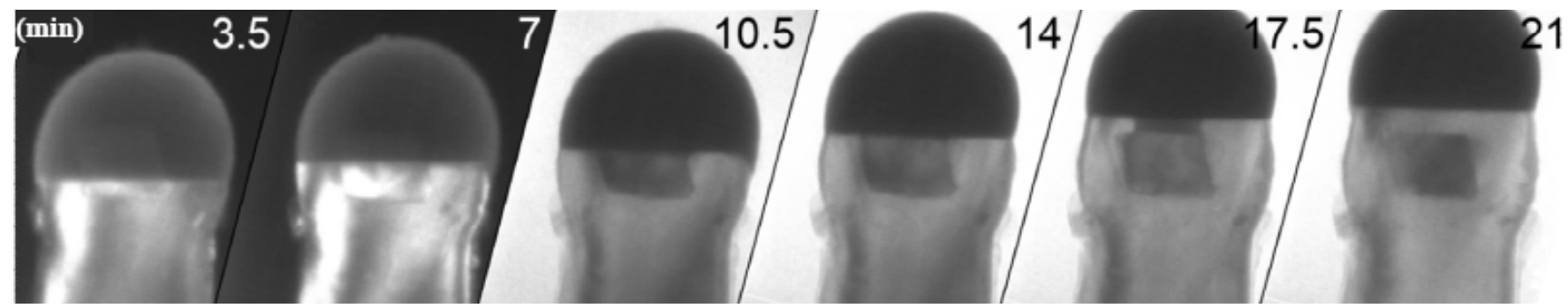

Figure 2. Continued growth of the nanowire in Figure 1 at $500^{\circ} \mathrm{C}$ and $5 \times 10^{-5}$ Torr $\mathrm{Si}_{2} \mathrm{H}_{6}$, showing the $\mathrm{NiSi}_{2}$ nanocrystal becoming embedded in the Si nanowire. Time shown in minutes since the final image of Figure 1. 